\title{
¿HAY QUE SANCIONAR LA PIRATERÍA? La protección de la propiedad intelectual
}

\author{
Nancy Matos, M. A. \\ INVESTIGADORA DOCTORAL ESADE-ESAN \\ GERENTE GENERAL DE ANDABAS S. R. L. \\ R.MATOS.R@esade.edu
}

\section{Resumen}

Desde la perspectiva de la conducta del consumidor, presenta un conjunto de reflexiones en torno a la protección de la propiedad intelectual. Pone énfasis en que la piratería es un tema complejo por lo difícil de establecer beneficios y daños y porque origina dilemas éticos entre los derechos de autor y los derechos de los demás. Luego de centrar el tema y señalar la magnitud que ha alcanzado la piratería de libros, software, discos y películas, analiza lo que significan las estrategias de segmentación de las empresas, así como el hecho de que gracias a la piratería los consumidores de menores recursos pueden acceder a mercados de los cuales, en otras circunstancias, estarían excluidos.

$\mathrm{L}$ a protección de la propiedad intelectual plantea a la sociedad dilemas legales, éticos y económicos que generan controversia; no son claras ni la necesidad de la protección ni las evidencias del daño ocasionado. En las líneas que siguen proponemos un conjunto de reflexiones que buscan analizar esta problemática desde la perspectiva del consumidor.

En primer lugar hay que precisar los alcances del término piratería que se utilizarán en este artículo. Según Shore y otros (2001), la piratería comprende dos categorías:
- Lifting, referida a la copia de software, películas, música, libros que el consumidor realiza para su uso personal.

- Piratería, referida a las copias para obtener ganancias ilícitas que realizan algunas organizaciones o personas.

De acuerdo con esta precisión, el lifting es una decisión de conducta del consumidor, mientras la piratería es una decisión de conducta empresarial -independientemente de que la empresa o agrupación involucrada en el acto de piratería sea formal o informal-. La oferta de produc- 
tos resultantes de actos de piratería inyecta al mercado un volumen adicional de productos en condiciones atractivas, que provocan la aceptación del consumidor.

También hay que distinguir entre piratería y falsificación. Esta última corresponde al ámbito de los bienes tangibles y consiste en la copia de un producto original con la intención de hacer ganancias, pero en este caso, a partir de un producto original, se debe aplicar ingeniería en reversa a fin de realizar una mejor o peor imitación. La falsificación requiere de una inversión inicial, además de incurrir en costos de reproducción y comercialización altos, lo que constituye una barrera a la entrada en este negocio.

La piratería, por su parte, corresponde a los bienes intangibles y no requiere aplicar tipo alguno de ingeniería, sino solamente utilizar la tecnología disponible; las copias son una perfecta reproducción del original; la inversión inicial (en adquisición de tecnología) es baja y los costos de reproducción son cercanos a cero.

\section{El alcance y real dimensión de la piratería}

De lo expuesto hasta ahora puede apreciarse que los actores involucrados en la piratería son varios: la empresa fabricante del original, los piratas que reproducen las copias, los consumidores que aceptan comprar originales o copias. Un actor adicional es el Estado, ya que las operaciones de falsificación y piratería se realizan al margen del mercado regulado y, por lo tanto, no pagan impuestos.

Veamos cuáles son los perjuicios que la piratería ocasiona.
Según datos de la Alianza Internacional de la Propiedad Intelectual ${ }^{1}$ (IIPA, 2003), se estima en 4,7 billones de dólares las pérdidas en derechos de propiedad intelectual ocasionadas a las empresas estadounidenses por la región Asia Pacífico, y en 257 millones de dólares las ocasionadas por los países del Grupo Andino. Estas pérdidas se concentran en ciertos tipos de bienes: música, software, películas y libros.

Como puede observarse en el cuadro adjunto, Bolivia presenta el índice más alto en piratería de películas: $100 \%$, mientras al Perú le corresponde el índice más alto en piratería musical: $98 \%$.

La forma de calcular estas cifras es muy importante. En el caso del software, por ejemplo, según explican Davenport y otros (2000), la estimación se basa en el supuesto de que cada computadora nueva que se vende debe estar acompañada de programas informáticos que la hagan funcionar. Luego, la diferencia entre las ventas esperadas y las ventas reales se atribuye a la piratería y, de este modo, el costo de las ventas no realizadas representa las pérdidas financieras de la industria del software.

El criterio parece razonable, pero ¿dónde quedan las estrategias de las empresas? Normalmente, éstas deciden el lanzamiento de productos al mercado so-

1. Se trata de una coalición que representa a las industrias de Estados Unidos afectadas por violaciones a los derechos de autor. Se basa en esfuerzos bilaterales y multilaterales para mejorar la protección internacional de las obras protegidas con derechos de autor. En el sitio web http://www.iipa.com se puede encontrar información general sobre esta institución, además de informes sobre la piratería en todo el mundo, por países y por temas. 


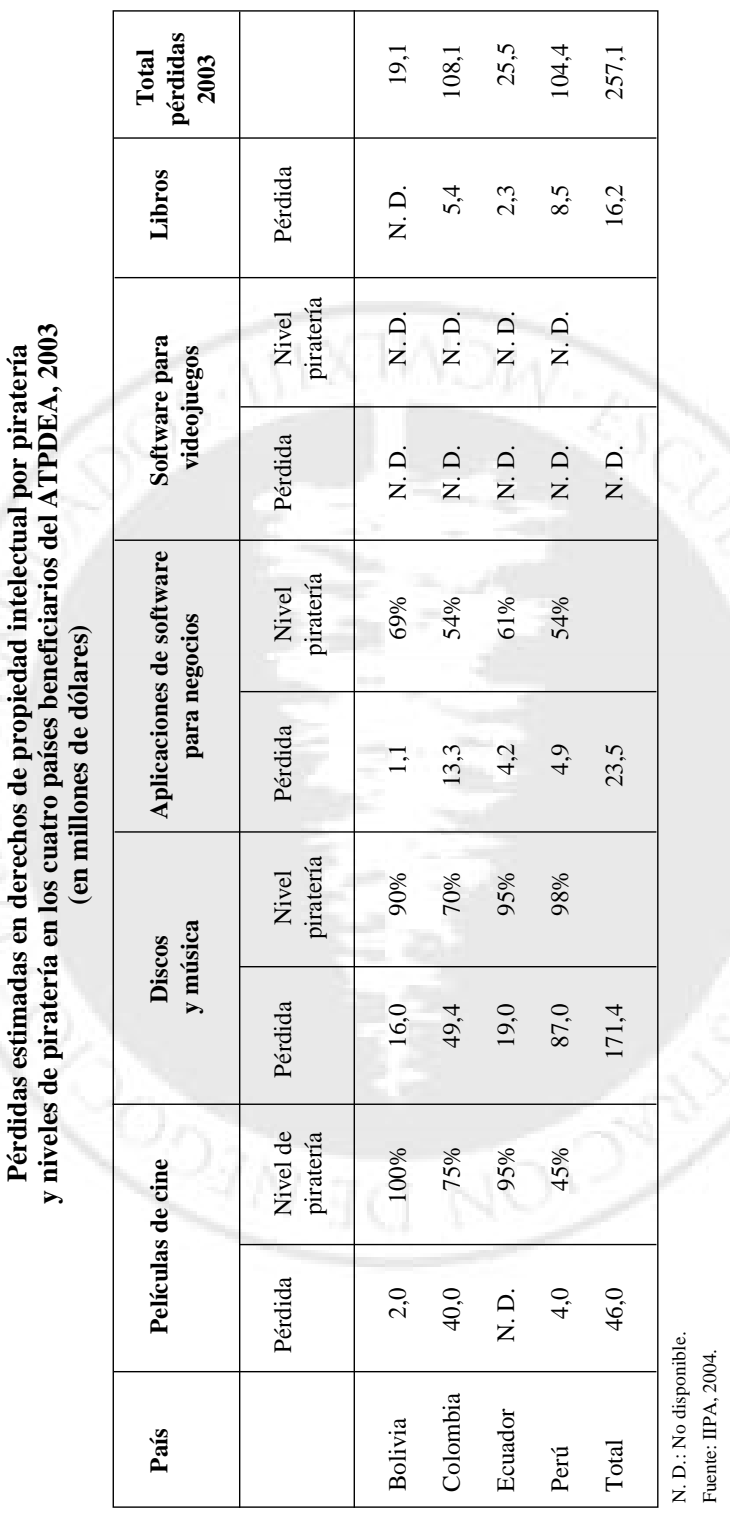


bre la base de la elección de un mercado (segmentación) donde pronostican un volumen de ventas a un precio determinado. Al realizar esta segmentación aceptan pagar las consecuencias de la misma; es decir, habrá consumidores que aceptarán comprar el producto y otros que considerarán no hacerlo, porque las condiciones no les parecen aceptables o porque su poder adquisitivo no se los permite.

La segmentación de mercados realizada por la empresa motiva que los competidores se acomoden en otros segmentos, o se disputen el volumen de ventas del mejor segmento, con lo que dejan abandonada una parte del mercado.

Tradicionalmente, estos mercados dejados de lado, que ofrecen pocos márgenes de ganancia, representan oportunidades para otros competidores que, por economías de escala o algún otro tipo de ventaja, pueden desarrollar una propuesta atractiva y al alcance de los consumidores descartados de la propuesta anterior. Así, entran en juego las leyes del mercado, conocidas como la oferta y la demanda.

Al margen de que la competencia sea leal o desleal, el proceso de segmentación ocurre porque el mercado no es homogéneo. Por lo tanto, es cuestionable el criterio utilizado para la estimación del perjuicio ocasionado por la piratería, al pretender que todo lo que se compra en el mercado y no se obtuvo del fabricante del producto original deba ser considerado como ventas perdidas por este fabricante, ya que en las condiciones ofrecidas por éste, esas ventas jamás se habrían producido. Sólo por citar un ejemplo, las librerías limeñas no pueden considerar como ventas perdidas los cientos de ejemplares pirateados de El código Da Vinci, vendi- dos a 15 soles. De hecho, quizá muy pocos de los consumidores de los ejemplares pirateados habrían estado dispuestos a comprar el libro original, cuyo precio bordea los 90 soles.

¿Qué ocurre en realidad? Un grupo considerable de consumidores compra productos pirata (copias) ofertados a precios bajos, al alcance de todos los bolsillos. Acceden así a un mercado del cual estarían excluidos de no mediar esta posibilidad. En consecuencia, los ofertantes pirata pueden considerarse especies de Robin Hood modernos; aclamados por unos y perseguidos por otros, que aparecen debido a la existencia de mercados insatisfechos, dejados de lado deliberadamente por las empresas al momento de segmentar sus mercados con el fin de asegurarse márgenes de ganancias razonables.

\section{Los derechos de propiedad intelectual y el mercado}

La piratería constituye una violación de los «derechos de propiedad»; derechos que se basan en la justicia natural, según señala el jurista Edmundo Pizarro (1974):

... nada es más propio de un hombre que el fruto de su pensamiento, sin embargo mientras que en el mundo material, se puede establecer dominio por apropiación, en el mundo de las ideas, no se puede impedir que la colectividad se aproveche de ellas.

Los derechos de propiedad evolucionan a «derechos de autor» con la aparición de la imprenta. El mismo Pizarro señala que es España en 1473, 1502 y 1763 el país que reconoce la necesidad de otor- 
gar privilegios exclusivos para imprimir al autor y sus herederos, con lo cual se concede el derecho moral (paternidad) y el derecho exclusivo (utilización económica).

La protección de los derechos de propiedad intelectual, o del campo de las ideas, surge de la dificultad para distinguir entre propiedad y dominio. En efecto, como no hay apropiabilidad no puede evitarse que la colectividad se aproveche de los productos del intelecto, a no ser que se establezca un derecho sobre los mismos. Hay, sin embargo, varias dificultades para la aplicación de este derecho porque vivimos en un mundo global. Es necesario que todos los países adopten la misma legislación.

Assafa Endeshaw (2003) refiere que la piratería es la paradoja de las leyes de propiedad intelectual, pues cuando un país adopta leyes que le son ajenas se ocasionan al menos tres problemas:

a) Se afecta el derecho soberano de cada nación a elegir la legislación en función de lo que quiere proteger o desarrollar.

b) Se genera un desequilibrio entre la legislación de derechos de propiedad y el desarrollo tecnológico de cada nación.

c) Se fomenta la violencia legal, al estar vigente una ley que no se cumple en las calles.

De otro lado la aplicación de estos derechos origina un dilema ético entre los derechos de autor y los derechos de los demás. La Teoría de la Colectividad señala que las obras no son propiedad de sus autores, sino que, por su destino, pertenecen al pueblo y, por tanto, no hay nada que proteger. Por el contrario, la Teoría de la Personalidad señala que la obra es una prolongación de la personalidad del autor y, por tanto, debe protegerse la relación entre el autor y su obra.

Concluimos que los derechos de propiedad intelectual afectados por la piratería son, por lo menos, cuestionables en su naturaleza y difíciles de proteger por carecer de apropiabilidad. Desde otra perspectiva, la aplicación de estos derechos trastoca las leyes del mercado, al permitir que una empresa se convierta en dominante no por el ofrecimiento de mejores productos, sino por emplear los mejores argumentos jurídicos para defender su derecho de propiedad intelectual.

En la figura 1 presentamos la dinámica del mercado que se establece según los criterios tradicionales de competencia (lo gráfico nos exime de mayores comentarios), mientras en la figura 2 presentamos el marco de competencia basado en los derechos de propiedad intelectual.

La figura 2 representa una división del mercado basada en las reglas de protección de la propiedad intelectual. La parte superior agrupa a las empresas que disponen de competencias claves específicas para proteger sus derechos, y a los consumidores que compran sus productos; la parte inferior reúne a los actores que consideran que las creaciones pertenecen a la colectividad.

Si bien la figura muestra dos tipos de consumidores, en la práctica puede tratarse de los mismos, cuya conducta difiere dependiendo de las circunstancias. ¿Qué circunstancias son aquellas que llevan a un 
CONGLMIDOF

FIFIMA

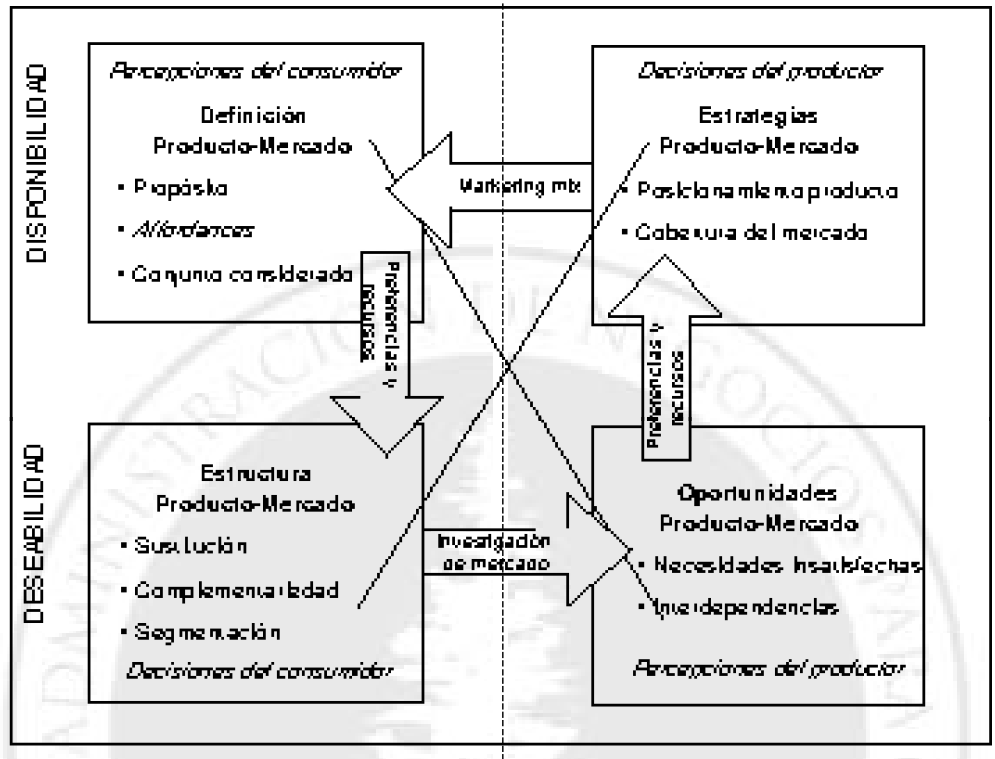

Tomado del Fambhwar v omos, 1 gas.

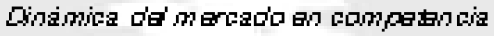

Figura?

consumidor a expresar una conducta de aceptación o rechazo frente a la piratería?

Pensamos que es necesario estudiar la respuesta a esta pregunta, antes de aplicar campañas de erradicación de la piratería para tratar de modificar esta conducta.

Los estudios sobre piratería basados en los aspectos económicos han tratado de establecer los beneficios o pérdidas que esta conducta ilegal puede causar a la sociedad. Utilizan modelos econométricos y se sustentan en un conjunto de premisas sobre conducta a partir de las cuales realizan sus cálculos.
Los estudios realizados, entre los que cabe mencionar especialmente los de Belleflamme (2002, 2003), concluyen que las firmas y la sociedad pierden sólo si la calidad de la copia es mala, pues las empresas carecerían de incentivos para ofrecer mayor calidad y dejarían de hacer innovación. El estudio de Chen y Png (2003) analiza el efecto de levantar barreras al copiado considerando la presencia de los que copian y de los que no copian; concluye que en estas circunstancias los que no copian se verían obligados a pagar un precio más alto que en una situación sin barreras al copiado, y los que copian lo harían con mayor razón, por lo 


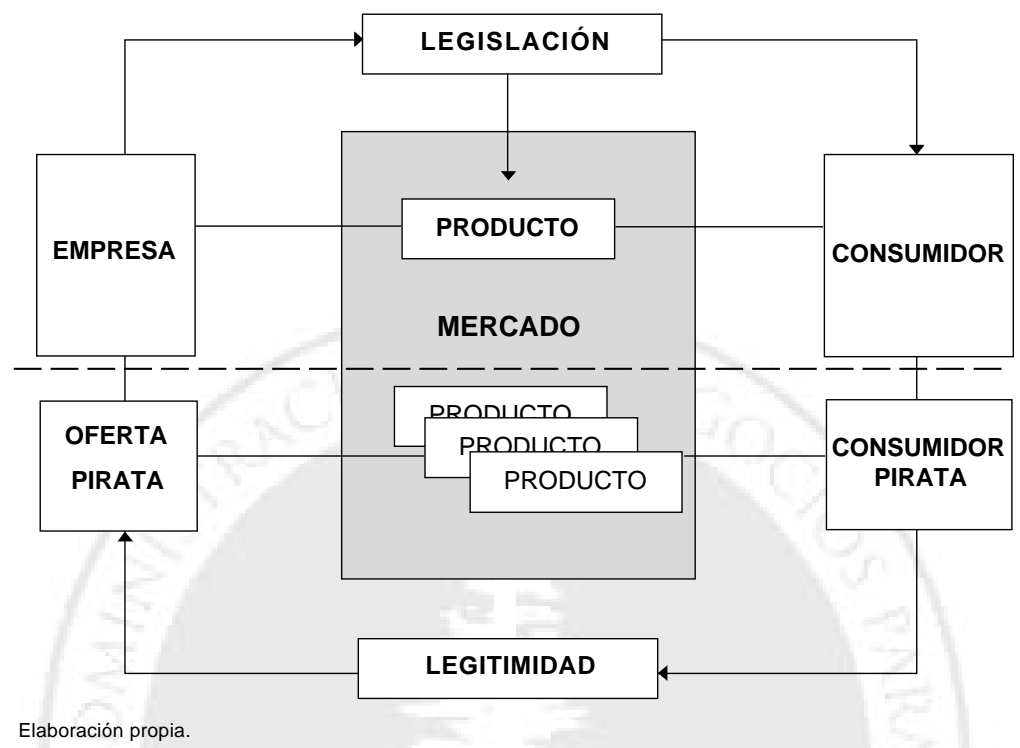

Competencia basada en derechos de propiedad intelectual Figura 2

que levantar barreras al copiado eleva los costos sociales y genera pérdida de bienestar a la sociedad, mientras que al bajar los precios, el efecto sería contrario.

De los estudios revisados, sólo uno logra probar que en el caso de que todos los consumidores tengan el mismo riesgo de ser sancionados, se podría controlar la piratería, pero esta situación, además de ser ideal, enfrenta a dos actores que deberían ser aliados: empresa y consumidor.

Para terminar, esta breve presentación ha buscado poner énfasis en que el problema de la piratería es complejo. Las autoridades que actualmente negocian el tratado de libre comercio, en el que el tema de la piratería y el condicionamiento a la aceptación de los derechos de propiedad está presente, deben tener en cuenta los aspectos paradójicos del problema y las tendencias relacionadas con éste.

En el caso de los productos digitales, la evolución tecnológica ha puesto al alcance del consumidor la posibilidad de copiar y obtener un producto de idéntica calidad que el original, gracias a tres características de estos bienes: no rivalidad (uso simultáneo por un número infinito de consumidores), no exclusión (una vez que alguien adquiere un producto, no hay 
medio de impedir que otro copie el mismo) y no ubicuidad (el producto se puede tener en cualquier lugar en forma instantánea).

Ahora nos enfrentamos no a un conjunto de empresas informales que copian productos y los venden en el mercado a consumidores de poca capacidad adquisitiva, sino a consumidores que han com- prado el original y desean compartir este producto con otros consumidores dispuestos a su vez a compartir los suyos; constituyendo una especie de clubes o asociaciones que se comunican por Internet.

Por ello es sumamente importante profundizar en el conocimiento del problema y, sobre todo, en la conducta del consumidor.

\section{Referencias bibliográficas}

ALIANZA INTERNACIONAL DE LA PROPIEDAD INTELECTUAL. http:// www.iipa.com (mayo 2004).

BARNEY, J. 1991. Firm resources and sustained competitive advantage. Journal of Management. Vol. 17, n. ${ }^{\circ}$ 1, págs. 99-120.

BAUMOL, W. J.; PANZER, J. C. y WILLIG, R. D. 1982. Contestable markets and the theory of industry structure. San Diego: Harcourt Brace Jovanovich.

BELLEFLAMME, P. 2003. Oligopolistic pricing of piratable information goods. Department of Economics, Queen Mary, University of London. www.econ. qmw.ac.uk/papers/wp463.htm.

2002. Pricing information goods in the presence of copying. Department of Economics, Queen Mary, University of London. Working Paper 463.

CHEN, Y. \& PNG, I. 2003. Information goods pricing and copyright enforcement: welfare analysis. Information Systems Research. Vol. 14, n. ${ }^{\circ}$ 1, Mar., págs. 107-123.
DAVENPORT y otros. 2000. Software piracy is not just economics anymore. Communications of the ACM. Dec., vol. 43, n. ${ }^{\circ} 12$, págs. 11-14.

ENDESHAW, A. 2003. The paradox of intellectual property lawmaking in the new millennium: universal templates as terms of surrender for non-industrial nations; piracy as an offshoot. Nanyang Technological University Singapore. Working Paper.

PIZARRO DÁVILA, Edmundo. 1974. Los bienes y derechos intelectuales. Lima: Arica.

RATNEHWAR, S.; SHOCKER, A.; COTTE, J. y SRIVASTAVA, R. 1999. Product, person, and purpose: putting the consumer back into theories of dynamic market behavior. Journal of Strategic Marketing. Vol. 7, págs. 191-208.

SHORE, B.; VENKATACHALAMA, A.; SOLORZANO, E.; BURN, J.; HASSAN, S. Z. \& JANCZEWSKI, L. J. 2001. Softlifting and piracy: behavior across cultures. Technology in Society. Vol. 23, n. ${ }^{\circ}$, págs. 563-581. 